\title{
A closed-form solution for a two-server heterogeneous retrial queue with threshold policy
}

\author{
TIEN VAN DO ${ }^{1,2, *}$, DÉNES PAPP ${ }^{2}$, RAM CHAKKA $^{3}$, JINTING WANG $^{4}$ and JÁNOS SZTRIK ${ }^{5}$ \\ ${ }^{1}$ Division of Knowledge and System Engineering for ICT, Faculty of Information Technology, Ton Duc Thang \\ University, Ho Chi Minh City, Vietnam \\ ${ }^{2}$ Department of Networked Systems and Services, Budapest University of Technology and Economics, H-117, \\ Magyar tudósok körútja 2, Budapest, Hungary \\ ${ }^{3}$ RGM College of Engineering and Technology (RGMCET), Nandyal, Kurnool District, Andhra Pradesh, India \\ ${ }^{4}$ Department of Mathematics, Beijing Jiaotong University, Beijing, China \\ ${ }^{5}$ Department of Informatics Systems and Networks, Faculty of Informatics, University of Debrecen, Debrecen, \\ Hungary \\ e-mail: dovantien@tdt.edu.vn; do@hit.bme.hu
}

MS received 1 June 2015; revised 14 March 2016; accepted 17 March 2016

\begin{abstract}
In this paper, we reconsider a two-server heterogeneous retrial queue with threshold policy. However, the computation time with the existing method is prohibitively large for certain values of the threshold parameter. Applying the spectral expansion method, we derive a closed-form expression for the eigenvalues and eigenvectors matrix that are needed to determine the steady-state distribution of a quasi-birth-death process describing the queue. As a result, the computation time for the performance measures does not depend on the threshold parameter.
\end{abstract}

Keywords. Retrial queue; two servers; threshold; spectral expansion; closed-form.

\section{Introduction}

Queueing theory has been applied to analyze the performance of telecommunication systems, modern information and communication technology (ICT) systems, productioninventory systems and manufacturing systems for long time [1-5]. Retrial queues where blocked customers may re-request for service after a certain timeout [1, 6-20] form a specific research topic in the queueing theory. It is worth mentioning that the impatient behaviour of customers can be modelled by retrial queues. Furthermore, a retrial mechanism can be also applied to control the access of resources in a certain system [21].

[22] considered a two-server heterogeneous retrial queue with threshold policy. They modelled the system as a quasi-birth-and-death (QBD) process with threshold dependent block-tridiagonal infinitesimal matrix and applied the general theory of matrix-geometric solutions. Thus, the computation of the rate matrix $R$ (the minimal non-negative solution to the matrix equation) is based on the iteration algorithm. However, their analysis procedure (see [22]) has limited applicability because the computational time significantly depends on the value of a threshold. To enhance the applicability of the two-server heterogeneous retrial queue with threshold policy for the analysis of practical systems such as ICT systems and manufacturing systems, we derive a closed-form solution for the steady-state probabilities. Therefore, the computational time needed to obtain the performance measures does not depend on the threshold, which is demonstrated by numerical results.

The rest of the paper is organized as follows. In section 2 , we describe a model. We present a mathematical derivation for the closed form solution in section 3 . Numerical results are presented in section 4 .

\section{A two-server heterogeneous retrial queue with threshold policy}

In this paper, we consider a retrial queue with two servers. The service time of a customer follows an exponential distribution with rate $\mu_{1}$ if the customer is served by the fast server and with rate $\mu_{2}$ if the customer is served by the slow server. Note that $\mu_{1}>\mu_{2}$. Customers arrive according to a Poisson process with rate $\lambda$. A customer (either arriving or retrial) gets service from the system if

- either the fast server is idle, or

*For correspondence 
- the fast server is busy, the slow server is idle and the number of customers in the orbit is above threshold $q_{2}$.

There is an orbit where customers are stored for the case when customers could not obtain service from the servers. According to the First Come First Serve (FCFS) principle, only the customer at the head of the orbit can retry for accessing servers. If the customer at the head of the orbit could not get service, he/she will return to the head of the orbit. The times between retrials (if the current retried customer is moved to the orbit when the orbit is not empty) follow the exponential distribution with parameter $\gamma$.

Let $J(t)$ denote the number of customers in the orbit at time $t$, and $I(t)$ describe the state of the servers as follows:

$I(t)=\left\{\begin{array}{cc}0 & \text { if two servers are idle } \\ 1 & \text { the fast server is busy and the slow server is idle } \\ 2 & \text { the fast server is idle and the slow server is busy } \\ 3 & \text { if two servers are busy }\end{array}\right.$

The entire system is described by Continuous Time Markov Chain (CTMC) $Y=\{I(t), J(t)\} \quad$ with state space $\{(i, j): 0 \leq i \leq 3, j \geq 0\}$. We denote the steady state probabilities by $\pi_{i, j}=\lim _{t \rightarrow \infty} \operatorname{Pr}(I(t)=i, J(t)=j)$, and introduce $\pi_{j}=\left(\pi_{0, j}, \pi_{1, j}, \pi_{2, j}, \pi_{3, j}\right)$.

The evolution of CTMC $Y$ is driven by the following transitions.

(a) $A_{j}(i, k)$ denotes a transition rate from state $(i, j)$ to state $(k, j) \quad(0 \leq i, k \leq 3 ; j=0,1, \ldots)$, which is caused by either the arrival of customers or the departure of customers after service. Matrix $A_{j}$ is defined as the matrix with elements $A_{j}(i, k)$.

(b) $B_{j}(i, k)$ represents one step upward transition rate from state $(i, j)$ to state $(k, j+1)(0 \leq i, k \leq 3 ; j=0,1, \ldots)$, which is due to the arrival of a customer when it could not obtain service. In the similar way, matrix $B_{j}(B)$ is defined with elements $B_{j}(i, k)$.

(c) $C_{j}(i, k)$ is the transition rate from state $(i, j)$ to state $(k, j-1)(0 \leq i, k \leq c ; j=1, \ldots)$, which is due to the successful retrial of a request from the orbit. Matrix $C_{j}$ $(\forall j \geq 1)$ is defined with elements $C_{j}(i, k)$.

Based on the operation rule, we obtain

$$
\begin{aligned}
A_{j} & =\left[\begin{array}{cccc}
0 & \lambda & 0 & 0 \\
\mu_{1} & 0 & 0 & 0 \\
\mu_{2} & 0 & 0 & \lambda \\
0 & \mu_{2} & \mu_{1} & 0
\end{array}\right] \text { for } 0 \leq j<q_{2} ; \\
A_{j} & =\left[\begin{array}{cccc}
0 & \lambda & 0 & 0 \\
\mu_{1} & 0 & 0 & \lambda \\
\mu_{2} & 0 & 0 & \lambda \\
0 & \mu_{2} & \mu_{1} & 0
\end{array}\right] \text { for } j \geq q_{2} ;
\end{aligned}
$$

$$
\begin{aligned}
& B_{j}=A_{01}=\left[\begin{array}{llll}
0 & 0 & 0 & 0 \\
0 & \lambda & 0 & 0 \\
0 & 0 & 0 & 0 \\
0 & 0 & 0 & \lambda
\end{array}\right] \text { for } 0 \leq j \leq q_{2}-1 ; \\
& B_{j}=A_{02}=\left[\begin{array}{llll}
0 & 0 & 0 & 0 \\
0 & 0 & 0 & 0 \\
0 & 0 & 0 & 0 \\
0 & 0 & 0 & \lambda
\end{array}\right] \text { for } j>q_{2}-1 ; \\
& C_{j}=A_{21}=\left[\begin{array}{llll}
0 & \gamma & 0 & 0 \\
0 & 0 & 0 & 0 \\
0 & 0 & 0 & \gamma \\
0 & 0 & 0 & 0
\end{array}\right] \text { for } 1 \leq j \leq q_{2} ; \\
& C_{j}=A_{22}=\left[\begin{array}{llll}
0 & \gamma & 0 & 0 \\
0 & 0 & 0 & \gamma \\
0 & 0 & 0 & \gamma \\
0 & 0 & 0 & 0
\end{array}\right] \text { for } j>q_{2} .
\end{aligned}
$$

Let us introduce $A_{00}=A_{0}-D^{A_{0}}-D^{B_{0}}, A_{10}=A_{1}-D^{A_{1}}-$ $D^{A_{01}}-D^{A_{21}}, A_{11}=A_{q_{2}}-D^{A_{q_{2}}}-D^{A_{02}}-D^{A_{21}}$ and $A_{12}=$ $A_{q_{2}}-D^{A_{q_{2}}}-D^{A_{22}}-D^{A_{02}}$, where $D^{Z}\left(Z=A_{j}, B_{j}, C_{j}\right)$ is a diagonal matrix whose diagonal element is the sum of all elements in the corresponding row of $Z$.

By equating the flow out and in each state, the balance equations can be expressed as below.

- In band 1, we have the balance equations

$$
\pi_{i-1} A_{01}+\pi_{i} A_{10}+\pi_{i+1} A_{21}=0, \quad 1 \leq i \leq q_{2}-1 .
$$

- The balance equations of band 2 is expressed as follows.

$$
\pi_{i-1} A_{02}+\pi_{i} A_{12}+\pi_{i+1} A_{22}=0, \quad i \geq q_{2}+1 .
$$

The boundary balance equations are

$$
\begin{gathered}
\pi_{0} A_{00}+\pi_{1} A_{21}=0, \\
\pi_{q_{2}-1} A_{01}+\pi_{q_{2}} A_{11}+\pi_{q_{2}+1} A_{22}=0 .
\end{gathered}
$$

\section{A closed-form solution}

Following [23], we obtain the expression for $\pi_{i}, 0 \leq i \leq q_{2}$, from Eq. (2)

$$
\pi_{i}=\sum_{k=1}^{4} a_{1, k} \psi_{1, k} x_{1, k}^{i}+\sum_{k=1}^{4} b_{1, k} \phi_{1, k} y_{1, k}^{q_{2}-1-i} \forall i=0, \ldots, q_{2}-1,
$$

where $a_{1, k}$ 's and $b_{1, k}$ 's are the coefficients to be determined, and $\left(x_{1, k}, \psi_{1, k}\right)(k=1,2,3,4)$ are the eigenvalue, lefteigenvector solution pairs of the matrix equations 


$$
\psi_{1,[}\left[A_{01}+A_{10} x_{1}+A_{21} x_{1,}^{2}\right]=0
$$

and $\left(y_{1, k}, \phi_{1, k}\right)(\mathrm{k}=1,2,3,4)$ are the eigenvalue, lefteigenvector solution pairs of the matrix equations

$$
\phi_{1,}\left[A_{21}+A_{10} y_{1,}+A_{01} y_{1,}^{2}\right]=0 .
$$

This means, we have to determine the appropriate eigenvalues and eigenvectors of the characteristic matrix polynomial

$$
\begin{aligned}
Q_{1}\left(x_{1}\right)= & A_{01}+x_{1} A_{10}+x_{1}^{2} A_{21} \\
= & {\left[\begin{array}{cc}
x_{1}(-\gamma-\lambda) & x_{1}^{2} \gamma+x_{1} \lambda \\
x_{1} \mu_{1} & \lambda+x_{1}\left(-\lambda-\mu_{1}\right) \\
x_{1} \mu_{2} & 0 \\
0 & x_{1} \mu_{2}
\end{array}\right.}
\end{aligned}
$$

The results are

$$
\begin{aligned}
& y_{1,1}=0, y_{1,2}=0, y_{1,3}=\frac{\gamma \mu_{1}}{\lambda(\gamma+\lambda)}, \\
& y_{1,4}=1, y_{1,5}=\frac{\omega-\sqrt{4 \gamma \mu_{1}\left(-\gamma \lambda-\lambda^{2}-\lambda \mu_{2}\right)+\omega^{2}}}{2\left(\gamma \lambda+\lambda^{2}+\lambda \mu_{2}\right)} \\
& y_{1,6}=\frac{\omega+\sqrt{4 \gamma \mu_{1}\left(-\gamma \lambda-\lambda^{2}-\lambda \mu_{2}\right)+\omega^{2}}}{2\left(\gamma \lambda+\lambda^{2}+\lambda \mu_{2}\right)},
\end{aligned}
$$

We can get eigenvalues $x_{1}$, by solving $\operatorname{Det}\left[Q_{1}\left(x_{1}\right)\right]=0$ as

$$
\begin{aligned}
& x_{1,1}=0, x_{1,2}=0, \\
& x_{1,3}=\frac{\omega-\sqrt{4 \gamma \mu_{1}\left(-\gamma \lambda-\lambda^{2}-\lambda \mu_{2}\right)+\omega^{2}}}{2 \gamma \mu_{1}}, \\
& x_{1,4}=\frac{\lambda(\gamma+\lambda)}{\gamma \mu_{1}}, x_{1,5}=1, \\
& x_{1,6}=\frac{\omega+\sqrt{4 \gamma \mu_{1}\left(-\gamma \lambda-\lambda^{2}-\lambda \mu_{2}\right)+\omega^{2}}}{2 \gamma \mu_{1}},
\end{aligned}
$$

where $\omega=\gamma \lambda+\lambda^{2}+\gamma \mu_{1}+\gamma \mu_{2}+2 \lambda \mu_{2}+\mu_{1} \mu_{2}+\mu_{2}^{2}$.

The eigenvectors are obtained as follows $\psi_{1,1}=[1,0,0,0]$, $\psi_{1,2}=[0,0,1,0], \quad \psi_{1,3}=\left[-1,-\frac{\gamma+\lambda+\mu_{2}}{\mu_{1}}, 1, \frac{\gamma+\lambda+\mu_{2}}{\mu_{1}}\right], \quad \psi_{1,4}=$ $\left[1, \frac{\gamma+\lambda}{\mu_{1}}, 0,0\right]$.

To obtain eigenvalues $y_{1}$, we solve $\operatorname{Det}\left[Q_{2}\left(y_{1}\right)\right]=0$, where and the eigenvectors are $\phi_{1,1}=[0,1,0,0], \phi_{1,2}=[0,0,0,1]$, $\phi_{1,3}=\left[1,-\frac{\gamma+\lambda+\mu_{2}}{\mu_{1}}, 1, \frac{\gamma+\lambda+\mu_{2}}{\mu_{1}}\right], \phi_{1,4}=\left[1, \frac{\gamma+\lambda}{\mu_{1}}, 0,0\right]$.

Following [24], the probability $\pi_{i}, i \geq q_{2}$, is given by

$$
\pi_{i}=\sum_{k=1}^{4} a_{2, k} \psi_{2, k} x_{2, k}^{i-q_{2}}, \quad \forall i \geq q_{2},
$$

where $\left(x_{2, k}, \psi_{2, k}\right)(k=1,2,3,4)$ are the eigenvalue, lefteigenvector solution pairs of the matrix equations

$$
\psi_{2,}\left[A_{02}+A_{12} x_{2},+A_{22} x_{2,}^{2}\right]=0 \text {. }
$$

Note that $\left|x_{2, k}\right|<1$ for $k=1,2,3,4$. This means, we have to determine the appropriate eigenvalues and eigenvectors of the characteristic matrix polynomial

$$
\begin{aligned}
Q_{2}\left(y_{1}\right) & =A_{21}+y_{1} A_{10}+y_{1}{ }^{2} A_{01} \\
& =\left[\begin{array}{cccc}
y_{1}(-\gamma-\lambda) & \gamma+y_{1} \lambda & 0 & 0 \\
y_{1} \mu_{1} & y_{1}^{2} \lambda+y_{1}\left(-\lambda-\mu_{1}\right) & 0 & 0 \\
y_{1} \mu_{2} & 0 & y_{1}\left(-\gamma-\lambda-\mu_{2}\right) & \gamma+y_{1} \lambda \\
0 & y_{1} \mu_{2} & y_{1} \mu_{1} & y_{1}^{2} \lambda+y_{1}\left(-\lambda-\mu_{1}-\mu_{2}\right)
\end{array}\right] .
\end{aligned}
$$




$$
\begin{aligned}
Q(x) & =A_{02}+x A_{12}+x^{2} A_{22} \\
& =\left[\begin{array}{cccc}
-(\gamma+\lambda) x & x(\lambda+\gamma x) & 0 & 0 \\
\mu_{1} x & -\left(\gamma+\lambda+\mu_{1}\right) x & 0 & x(\lambda+\gamma x) \\
\mu_{2} x & 0 & -\left(\gamma+\lambda+\mu_{2}\right) x & x(\lambda+\gamma x) \\
0 & \mu_{2} x & \mu_{1} x & \lambda-\left(\lambda+\mu_{1}+\mu_{2}\right) x
\end{array}\right] .
\end{aligned}
$$

It yields

$$
\begin{aligned}
\operatorname{Det}[Q(x)]= & x^{3}(x-1)\left[\lambda\left((\gamma+\lambda)^{2}+\gamma \mu_{1}\right)\left(\gamma+\lambda+\mu_{2}\right)\right. \\
& -\gamma\left(\mu_{1}\left(\gamma^{2}+2 \lambda^{2}+\gamma\left(3 \lambda+\mu_{1}\right)\right)\right. \\
& +\left((\gamma+\lambda)^{2}+2(\gamma+2 \lambda) \mu_{1}+\mu_{1}^{2}\right) \mu_{2} \\
& \left.\left.+\left(\gamma+\lambda+\mu_{1}\right) \mu_{2}^{2}\right) x+\gamma^{2} \mu_{1}\left(\mu_{1}-\mu_{2}\right) x^{2}\right] \\
& =x^{3}(x-1)\left[\omega_{0}-\omega_{1} x+\omega_{2} x^{2}\right]
\end{aligned}
$$

where

$$
\begin{aligned}
\omega_{0}= & \lambda\left((\gamma+\lambda)^{2}+\gamma \mu_{1}\right)\left(\gamma+\lambda+\mu_{2}\right), \\
\omega_{1}= & \gamma\left(\mu_{1}\left(\gamma^{2}+2 \lambda^{2}+\gamma\left(3 \lambda+\mu_{1}\right)\right)\right. \\
& +\left((\gamma+\lambda)^{2}+2(\gamma+2 \lambda) \mu_{1}+\mu_{1}^{2}\right) \mu_{2} \\
& \left.+\left(\gamma+\lambda+\mu_{1}\right) \mu_{2}^{2}\right), \\
\omega_{2}= & \gamma^{2} \mu_{1}\left(\mu_{1}-\mu_{2}\right) .
\end{aligned}
$$

As a consequence, $\operatorname{Det}[Q(x)]$ has three zero roots $\left(x_{2,1}=x_{2,2}=x_{2,3}=0\right)$, one root equal to 1 . In addition,

- $\operatorname{Det}[Q(x)]$ has one $\operatorname{root} x_{2,4}^{*}=\frac{\gamma^{2} \lambda+2 \gamma \lambda^{2}+\lambda^{3}+\gamma \lambda \mu_{2}}{\gamma \mu_{2}\left(2 \gamma+3 \lambda+2 \mu_{2}\right)}$ if $\mu_{1}=\mu_{2}$.

- $\operatorname{Det}[Q(x)]$ has two roots $x_{2,4}=\frac{\omega_{1}-\sqrt{\omega_{1}^{2}-4 \omega_{0} \omega_{2}}}{2 \omega_{2}}$ and $x_{2,5}=\frac{\omega_{1}+\sqrt{\omega_{1}^{2}-4 \omega_{0} \omega_{2}}}{2 \omega_{2}}$ if $\mu_{1} \neq \mu_{2}$.

Note that the eigenvalues of Eq. (13) are the roots of $\operatorname{Det}[Q(x)]$. Following the same argument as in [8], if the QBD process is ergodic, $Q(x)$ should have four eigenvalues inside the unit circle. As a consequence, $\left|x_{2,4}^{*}\right|<1$ (for $\mu_{1}=\mu_{2}$ ) and $\left|x_{2,4}\right|<1$ (for $\mu_{1} \neq \mu_{2}$ ). In what follows, we also use $x_{2,4}$ to refer to $x_{2,4}^{*}$ when $\mu_{1}=\mu_{2}$.

It is easy to check that independent left-eigenvectors corresponding to three null-eigenvalues are $\psi_{2,1}=[1,0,0,0], \psi_{2,2}=[0,1,0,0], \ldots, \psi_{2,3}=[0,0,1,0]$.

Let $\psi_{2,4}=\left[\psi_{2,4,1}, \psi_{2,4,2}, \psi_{2,4,3}, 1\right]$ be the eigenvector corresponding to $x_{2,4}$. Utilizing $\psi_{2,4} Q\left(x_{2,4}\right)=0$, we get

$$
\begin{gathered}
\psi_{2,4,1}=\frac{2 \gamma \mu_{1} \mu_{2}+2 \lambda \mu_{1} \mu_{2}+\mu_{1}^{2} \mu_{2}+\mu_{1} \mu_{2}^{2}}{\left(\gamma+\lambda+\mu_{2}\right)\left(\gamma^{2}+2 \gamma \lambda+\lambda^{2}+\gamma \mu_{1}-\gamma \mu_{1} x_{2,4}\right)} \\
\psi_{2,4,2}=-\frac{-\gamma^{2} \mu_{2}-2 \gamma \lambda \mu_{2}-\lambda^{2} \mu_{2}-\lambda \mu_{1} \mu_{2}-\gamma \mu_{2}^{2}-\lambda \mu_{2}^{2}-\gamma \mu_{1} \mu_{2} x_{2,4}}{\left(\gamma+\lambda+\mu_{2}\right)\left(\gamma^{2}+2 \gamma \lambda+\lambda^{2}+\gamma \mu_{1}-\gamma \mu_{1} x_{2,4}\right)} \\
\psi_{2,4,3}=\frac{\mu_{1}}{\gamma+\lambda+\mu_{2}} .
\end{gathered}
$$

Besides (2), (3), (4) and (5) the normalization equation can be used to determine the coefficients:

$$
\sum_{i=0}^{\infty} \pi_{i} e=\sum_{i=0}^{q_{2}-1} \pi_{i} e+\sum_{i=q_{2}}^{\infty} \pi_{i} e=1
$$

Since

$$
\begin{aligned}
\sum_{i=0}^{q_{2}-1} \pi_{i} e= & \sum_{k=1}^{4} a_{1, k} \Psi_{1, k} \frac{-1+x_{1, k}^{q_{2}}}{-1+x_{1, k}} e \\
& +\sum_{k=1}^{4} b_{1, k} \Phi_{1, k} \frac{-1+y_{1, k}^{q_{2}}}{-1+y_{1, k}} e \\
\sum_{i=q_{2}}^{\infty} \pi_{i} e= & \sum_{k=1}^{4} a_{2, k} \Psi_{2, k} \frac{1}{1-x_{2, k}} e
\end{aligned}
$$

the solution for coefficients are

$$
\begin{aligned}
a_{1,1} & =G_{11} a_{1,3}, a_{1,2}=\frac{1}{\lambda+\mu_{2}} \gamma\left(1-\tau_{6} y_{1,3} \mu_{1} G_{12}\right) a_{1,3}, \\
a_{1,4} & =G_{14} a_{1,3}, b_{1,1}=0, b_{1,2}=0, b_{1,3}=G B_{13} a_{1,3}, \\
b_{1,4} & =G B_{14} a_{1,3}, a_{2,1}=G_{21} a_{1,3}, a_{2,2}=G_{22} a_{1,3}, a_{2,3}=0, \\
a_{2,4} & =G_{24} a_{1,3}, \\
a_{1,3} & =1 /\left(G_{11}+\frac{\gamma\left(1-G_{12} \mu_{1} \tau_{6} y_{1,3}\right)}{\lambda+\mu_{2}}+G_{14} \tau_{13} \tau_{14}+G_{14} \tau_{13}\right. \\
& \left.+G_{21}+G_{22}+\frac{G_{24}\left(-\tau_{1}+\tau_{2}-1\right)}{x_{2,4}-1}+G B_{14}\left(\tau_{14}+1\right)\right),
\end{aligned}
$$


where

$$
\begin{aligned}
x_{1,3}= & 1 /\left(2 \gamma \mu_{1}\right)\left(\gamma \lambda+\lambda^{2}+\gamma \mu_{1}+\gamma \mu_{2}+2 \lambda \mu_{2}+\mu_{1} \mu_{2}+\mu_{2}^{2}\right. \\
& \left.-\sqrt{-4 \gamma \lambda \mu_{1}\left(\gamma+\lambda+\mu_{2}\right)+\left(\lambda^{2}+2 \lambda \mu_{2}+\mu_{2}\left(\mu_{1}+\mu_{2}\right)+\gamma\left(\lambda+\mu_{1}+\mu_{2}\right)\right)^{2}}\right), \\
y_{1,3}= & 1 /\left(2 \lambda\left(\gamma+\lambda+\mu_{2}\right)\right)\left(\gamma \lambda+\lambda^{2}+\gamma \mu_{1}+\gamma \mu_{2}+2 \lambda \mu_{2}+\mu_{1} \mu_{2}+\mu_{2}^{2}\right. \\
& -\sqrt{\left.-4 \gamma \lambda \mu_{1}\left(\gamma+\lambda+\mu_{2}\right)+\left(\lambda^{2}+2 \lambda \mu_{2}+\mu_{2}\left(\mu_{1}+\mu_{2}\right)+\gamma\left(\lambda+\mu_{1}+\mu_{2}\right)\right)^{2}\right),} \\
x_{2,4}= & 1 /\left(2 \gamma^{2} \mu_{1}\left(\mu_{1}-\mu_{2}\right)\right)\left(\gamma^{3} \mu_{1}+3 \gamma^{2} \lambda \mu_{1}+2 \gamma \lambda^{2} \mu_{1}+\gamma^{2} \mu_{1}^{2}+\gamma^{3} \mu_{2}+2 \gamma^{2} \lambda \mu_{2}\right. \\
& +\gamma \lambda^{2} \mu_{2}+2 \gamma^{2} \mu_{1} \mu_{2}+4 \gamma \lambda \mu_{1} \mu_{2}+\gamma \mu_{1}^{2} \mu_{2}+\gamma^{2} \mu_{2}^{2}+\gamma \lambda \mu_{2}^{2}+\gamma \mu_{1} \mu_{2}^{2} \\
& -\left(\gamma ^ { 2 } \left(-4 \lambda \mu_{1}\left(\gamma^{2}+\lambda^{2}+\gamma\left(2 \lambda+\mu_{1}\right)\right)\left(\mu_{1}-\mu_{2}\right)\left(\gamma+\lambda+\mu_{2}\right)+\left(\gamma^{2}\left(\mu_{1}+\mu_{2}\right)\right.\right.\right. \\
& +\mu_{1} \mu_{2}\left(\mu_{1}+\mu_{2}\right)+\lambda^{2}\left(2 \mu_{1}+\mu_{2}\right)+\lambda \mu_{2}\left(4 \mu_{1}+\mu_{2}\right) \\
& \left.\left.\left.+\gamma\left(\left(\mu_{1}+\mu_{2}\right)^{2}+\lambda\left(3 \mu_{1}+2 \mu_{2}\right)\right)\right)^{2}\right)\right) \\
\tau_{1}= & \left(\left(-1+x_{2,4}\right) \lambda+x_{2,4}\left(\mu_{1}+\mu_{2}\right)\right) /\left(x_{2,4}\left(x_{2,4} \gamma+\lambda\right)\right), \\
\tau_{2}= & \left(\mu_{2}+\left(\gamma+\lambda+\mu_{1}\right)\left(\mu_{1} /\left(\gamma+\lambda+\mu_{2}\right)-\tau_{1}\right)\right) /\left(x_{2,4} \gamma+\lambda\right), \\
\tau_{3}= & \left(\lambda^{q_{2}-1}(\gamma+\lambda)^{q_{2}}\right) /\left(\gamma^{q_{2}-1} \mu_{1}^{q_{2}}\right) \\
\tau_{4}= & x_{1,3}^{q_{2}-1}\left(\gamma+\lambda+\mu_{2}\right), \\
\tau_{5}= & \left(\lambda^{q_{2}-1}(\gamma+\lambda)^{q_{2}-1}\right) /\left(\gamma^{q_{2}-1} \mu_{1}^{q_{2}-1}\right), \\
\tau_{6}= & y_{1,3}^{q_{2}-2} \\
\tau_{7}= & \mu_{1} /\left(\gamma+\lambda+\mu_{2}\right), \\
\tau_{8}= & x_{1,3}^{q_{2}-2} \\
\tau_{9}= & \mu_{1}^{q_{2}-2}, \\
\tau_{10}= & \left(\lambda^{q_{2}-2}(\gamma+\lambda)^{q_{2}-1}\right) / \gamma^{q_{2}-2}, \\
\tau_{11}= & \left(\lambda^{q_{2}}(\gamma+\lambda)^{q_{2}}\right) /\left(\gamma^{q_{2}} \mu_{1}^{q_{2}}\right), \\
\tau_{12}= & \left(\gamma+\lambda+\mu_{1}\right) / \mu_{1}, \\
\tau_{13}= & \left(-1+\tau_{11}\right) /\left(-1+(\lambda(\gamma+\lambda)) /\left(\gamma \mu_{1}\right)\right), \\
\tau_{14}= & (\gamma+\lambda) / \mu_{1}, \\
& \\
&
\end{aligned}
$$

and $G_{11}, G_{12}, G_{14}, G_{21}, G B_{22}, G_{24}, G B_{13}$ and $G B_{14}$ are expressed as long equations in the e-companion [25].

\section{Numerical results}

\subsection{Comparing computational methods}

We compare the execution times of a method presented in [22] with our closed-form solution with parameters $\lambda=2.2, \gamma=16.5, \mu_{1}=2.6$ and $\mu_{2}=0.3$. Mathematica scripts were written and were executed in a machine with Intel Xeon E5410 $2.33 \mathrm{GHz}$ processor to produce results.

The execution times vs $q_{2}$ are depicted in figure 1 . The execution times of a closed-form solution are independent

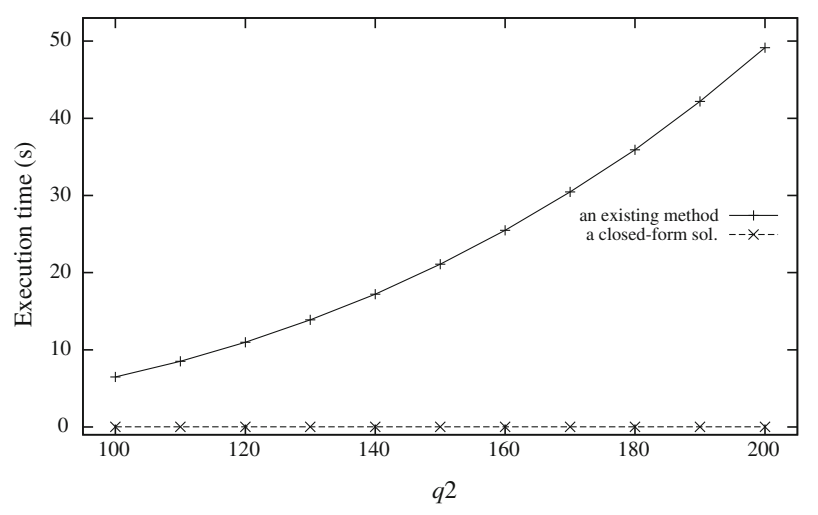

Figure 1. Comparison of computation methods. 
from $q_{2}$ (see figure 2), while the execution times of a method by [22] rapidly increase. We get results for $q_{2}=$ $4 \times 10^{4}$ when no result can be obtained with a method presented in [22].

Figure 3 shows the average time spent by customers in the system for the $q_{2}$ interval from 10 to 700 when the first server is $(60,80,100,120)$-fold faster than the second server. It can be observed that the average system time of customers can be minimized by the appropriate choice of $q_{2}$. It is also observed from table 1 that $r=\mu_{1} / \mu_{2}$ has a small impact on the choice of $q_{2}$.

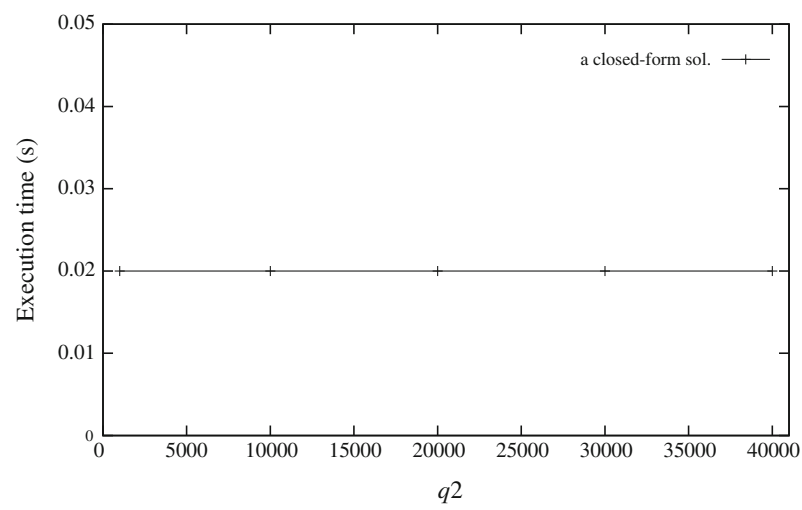

Figure 2. The execution times of a new method.

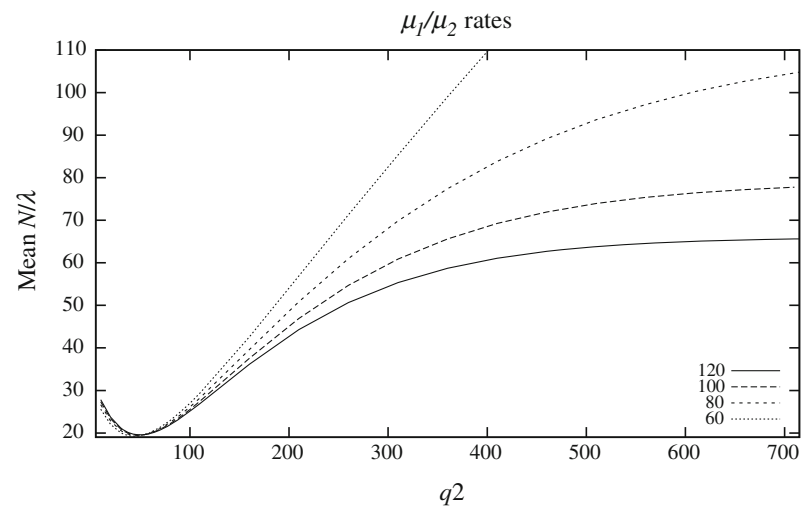

Figure 3. The average system time vs $r=\mu_{1} / \mu_{2}$.

Table 1. $q_{2}$ value when the average system time has the lowest value for $r=\mu_{1} / \mu_{2}$.

\begin{tabular}{lcc}
\hline$r=\mu_{1} / \mu_{2}$ & $q_{2}$ & The average system time \\
\hline 60 & 44 & 19.259 \\
80 & 46 & 19.4357 \\
100 & 48 & 19.5188 \\
120 & 50 & 19.5629 \\
\hline
\end{tabular}

\section{Conclusions}

We have provided closed-form equations for the steady state probabilities and the performance measures of a twoserver retrial queue with the threshold policy. Numerical results clearly demonstrate the advantage of the new method over the existing method.

The operation mode considered in this paper can be used to model a practical situation related to the application of two physical servers to provide IT service. The investigation will be our future work.

\section{References}

[1] Artalejo J R and Gómez-Corral A 2008 Retrial queueing systems. Springer-Verlag, Berlin Heidelberg

[2] Kleinrock L 1975 Queueing systems. Vol I Theory. John Wiley \& Sons, Inc

[3] Ram R and Viswanadham N 1990 Stochastic analysis of versatile workcentres. Sadhana 15(4-5): 301-317

[4] Viswanadham N and Srinivasa Raghavan N 1997 Flexibility in manufacturing enterprises. Sadhana 22(2): 135-163

[5] Viswanadham N 2000 Analysis of manufacturing enterprises. Springer

[6] Artalejo J R 2010 Accessible bibliography on retrial queues: Progress in 2000-2009. Math. Comp. Model. 51(9-10): 1071-1081

[7] Dimitriou I 2015 Performance modeling of cellular systems with finite processor $\mathrm{S}$ haring queues in random environment, guard policy and flex retrial users. In: Gribaudo M, Manini D and Remke A (Eds) Analytical and stochastic modelling techniques and applications. Springer International Publishing, 43-58

[8] Do T V and Chakka R 2010 An efficient method to compute the rate matrix for retrial queues with large number of servers. Appl. Math. Lett. 23: 638-643

[9] Do T V, Do N H and Zhang J 2013 An enhanced algorithm to solve multiserver retrial queueing systems with impatient customers. Comput. Ind. Eng. 65(4): 719-728

[10] Do T V 2010 A new computational algorithm for retrial queues to cellular mobile systems with guard channels. Comput. Ind. Eng. 59(4): 865-872

[11] Do T V 2011 Solution for a retrial queueing problem in cellular networks with the fractional guard channel policy. Math. Comp. Model. 53(11-12): 2058-2065

[12] Dragieva V I 2015 Steady state analysis of the M/G/1//N queue with orbit of blocked customers. Ann. Oper. Res. doi:10.1007/s10479-015-2025-z

[13] Falin G I and Templeton J G C 1997 Retrial queues. Chapman \& Hall, London

[14] Gao S, Wang J and Do T V 2015 A repairable retrial queue under bernoulli schedule and general retrial policy. Ann. Oper. Res. doi:10.1007/s10479-015-1885-6

[15] Gharbi N, Nemmouchi B, Mokdad L and Ben-Othman J 2014 The impact of breakdowns disciplines and repeated attempts on performances of small cell networks. J. Comput. Sci. 5(4): 633-644 
[16] Vasiliadis G 2016 Transient analysis of a finite source discrete-time queueing system using homogeneous Markov system with state size capacities (hms/c). Commun. Stat. Theory Methods 45(5): 1403-1423

[17] Wang J and Zhang P 2009 A discrete-time retrial queue with negative customers and unreliable server. Comput. Ind. Eng. 56(4): 1216-1222

[18] Wang J, Wang N and Alfa A S 2013 Discrete-time GI/G/1 retrial queues with time-controlled vacation policies. Acta Math. Appl. Sin., English Ser. 29(4): 689-704

[19] Wang J, Gao S and Do T V 2016 Performance analysis of a two-node computing cluster. Comput. Ind. Eng. 93: 227-235

[20] Zhang Y, Wang J and Wang F 2016 Equilibrium pricing strategies in retrial queueing systems with complementary services. Appl. Math. Model. doi:10.1016/j.apm.2016.01.029

[21] Choi B D, Shin Y W and Ahn W C 1992 Retrial queues with collision arising from unslotted CSMA/CD protocol. Queueing Syst. 11(4): 335-356
[22] Efrosinin D and Sztrik J 2011 Performance analysis of a twoserver heterogeneous retrial queue with threshold policy. Quality Technol. Quant. Manag. 8(3): 211-236

[23] Chakka R 1995 Performance and reliability modelling of computing systems using spectral expansion. Ph.D. thesis, University of Newcastle upon Tyne (Newcastle upon Tyne)

[24] Mitrani I and Chakka R 1995 Spectral expansion solution for a class of Markov models: Application and comparison with the matrix-geometric method. Performance Eval. 23(3): 241-260

[25] Do T V, Papp D, Chakka R, Wang J and Sztrik J The supplementary materials. [Online] available from http://www. hit.bme.hu/ do/eqs_br6, 2016 